\title{
Modeling of piezoelectric actuator's hysteresis and its effect on the control accuracy of a LEO-to-GEO laser-communication for a small satellite
}

Phong Xuan Do, Ryo Suzumoto, Alberto CarrascoCasado, Florian Moll, Takayuki Hosonuma, et al.

Phong Xuan Do, Ryo Suzumoto, Alberto Carrasco-Casado, Florian Moll, Takayuki Hosonuma, Morio Toyoshima, Shinichi Nakasuka, "Modeling of piezoelectric actuator's hysteresis and its effect on the control accuracy of a LEO-to-GEO laser-communication for a small satellite," Proc. SPIE 11852, International Conference on Space Optics - ICSO 2020, 118523B (11 June 2021); doi: 10.1117/12.2599541

SPIE Event: International Conference on Space Optics - ICSO 2021, 2021, Online Only 


\section{International Conference on Space Optics-ICSO 2020}

Virtual Conference

30 March-2 April 2021

Edited by Bruno Cugny, Zoran Sodnik, and Nikos Karafolas
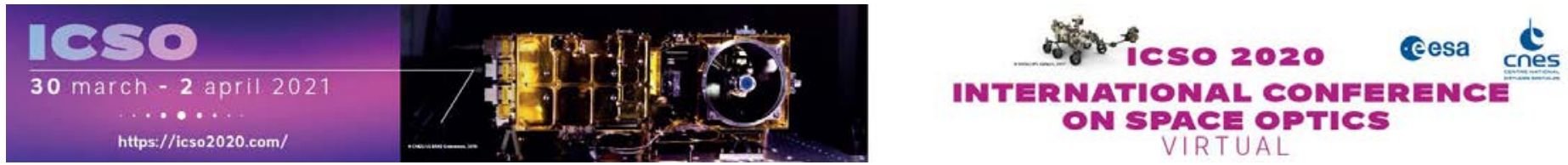

\section{Modeling of piezoelectric actuator's hysteresis and its effect on the control accuracy of a LEO-to-GEO laser-communication for a small satellite}

\section{Cesa isopoceatings ecnes}




\title{
Modeling of piezoelectric actuator's hysteresis and its effect on the control accuracy of a LEO-to-GEO laser-communication for a small satellite
}

\author{
Phong Xuan Do ${ }^{\mathrm{a}}$, Ryo Suzumoto ${ }^{\mathrm{a}}$, Alberto Carrasco-Casado ${ }^{\mathrm{b}}$, Florian Moll ${ }^{\mathrm{c}}$, Takayuki \\ Hosonuma $^{\mathrm{a}}$, Morio Toyoshima ${ }^{\mathrm{b}}$, and Shinichi Nakasuka ${ }^{\mathrm{a}}$ \\ aDepartment of Aeronautics and Astronautics, University of Tokyo, Tokyo, Japan \\ ${ }^{b}$ Space Communications Laboratory, National Institute of Information and Communications \\ Technology, Tokyo, Japan \\ ${ }^{\mathrm{c}}$ Institute of Communication and Navigation, DLR, Oberpfaffenhofen, Germany
}

\begin{abstract}
Compared to conventional large satellites in the past, small satellite classes (less than $150 \mathrm{~kg}$ ) show their advantages for mass production, such as short time and low cost for development and launch, to cope with the demand for emerging missions that require a sufficient number of satellites in orbit. However, the traditional communication method, in which a low earth orbit (LEO) small satellite sends data to a ground station using radio frequency, has several disadvantages. Firstly, the limitation of radio-frequency bandwidth leads to a low data rate and difficulty in getting a frequency license. Secondly, there is a significant delay during which data cannot be sent to the ground due to lacking a line of sight between the LEO satellite and the ground station. Additionally, the duration time for the small satellite to communicate with the ground station is just less than 10 minutes approximately. To resolve the above issues, we investigate the case that a less-than-150-kg satellite carries out a laser communication link from LEO to a satellite in geostationary orbit (GEO). Due to the constraints of size, weight, and power (SWaP), traditional bulky LEO-GEO relay systems cannot be applied for the small satellite. However, using the combination of the satellite body pointing and a piezo Fast-Steering Mirror (FSM), which reduces the SWaP considerably, makes it feasible that the LEO-to-GEO communication can be implemented in a small satellite for the first time. While utilizing laser communication can increase the data rate, the relay communication via the GEO satellite helps the small satellite to extend the communication duration significantly. Moreover, since there is a line of sight between the two terminals in any of about 15 orbits per day of the LEO satellite, data taken by the small satellite can be downloaded to the ground via the GEO one in almost real time. This research aims at investigating and proving the feasibility of a small satellite to transmit a laser communication link to its GEO counterpart. In this paper, we describe the LEO-to-GEO laser communication of the small satellite with a study of pointing-budget and link-budget analysis. Furthermore, a hardware-based simulation of the fine control mechanism is conducted. The hysteresis that affects severely to the piezo mechanism, and hence, the final control accuracy, is modeled accurately and its effect is shown.
\end{abstract}

Keywords: small satellite, laser communication, LEO, relay, GEO

\section{INTRODUCTION}

Over the past few years, many remote-sensing applications require high-frequency-observing data produced by LEO satellites. To cope with this demand, constellations formed by a sufficient number of satellites are required. While it is infeasible for traditional large and costly satellites to fulfill the above requirement, the 100-kg class and micro/nano-satellites have been emerging to be a good alternative. These small satellites that require low cost and short time for development and launch show their potential for mass production and thus, make it feasible to accomplish the above missions. Additionally, with the advancement of technologies, small satellites have improved their capabilities to accomplish advanced missions. Until now, a 100-kg class satellite can take

Further author information: (Send correspondence to Phong Xuan Do)

Phong Xuan Do: E-mail: do-xuan-phong663@g.ecc.u-tokyo.ac.jp 
Table 1: Past LEO-GEO laser communication projects.

\begin{tabular}{|l|c|c|c|c|}
\hline Year & LEO satellite & $\begin{array}{c}\text { LEO satellite's } \\
\text { mass }(\mathbf{k g})\end{array}$ & $\begin{array}{c}\text { LEO-to-GEO link } \\
\text { data rate (Mbps) }\end{array}$ & GEO satellite \\
\hline 2001 & SPOT-4 & 2550 & 50 & ARTEMIS \\
\hline 2005 & OICETS & 570 & 50 & ARTEMIS \\
\hline 2017 & SENTINEL 1A & $\approx 2300$ & 1800 & EDRS-A \\
\hline
\end{tabular}

high-quality images of the Earth's surface at any time and in any weather conditions using the synthetic-apertureradar technology, ${ }^{1}$ which is used to be applicable for traditional large satellites. Because of these above facts, 100-kg class and smaller satellites have become a significant tool to provide always-up-to-date Earth-observing data to users. Apparently, in the above missions, it is crucial that data taken by small satellites must be sent to ground quickly to free up on-board memories and increase the efficiency of data utilization. However, the traditional satellite-to-ground communication method is limited in terms of duration for communication and responsiveness of data delivery. Moreover, with the rapidly increasing of the number of satellites launched, the radio frequency spectrum has become more congested. Therefore, LEO-to-GEO laser communication for smallsatellite classes are promising for future missions. Compared to satellite-to-ground communication, the method of relaying data through GEO satellites shows its merits in expanding the communication window and reducing the delay of data delivery.

Over the past two decades, LEO-GEO laser communication relay was implemented in several projects. ${ }^{2-4}$ Since the first demonstration of the LEO SPOT-4 and GEO ARTEMIS satellites, the technology has been advancing rapidly (see Tab. 1). Until recently, the LEO SENTINEL satellites have successfully communicated with the GEO satellites of the European Data Relay System at an average data rate of 1.8 Gbps. Nevertheless, there has not been a study on using the LEO-GEO laser communication model for 100-kg class or smaller satellites. To fill this gap, we study the concept in which a $100-\mathrm{kg}$ class satellite transmits data to a GEO satellite via a laser link. In this paper, by investigating state-of-the-art technologies in laser communication for small satellites, we conduct a trade-off study to propose a practical link budget for both initial and communication phases. To realize the stringent pointing requirement derived from the link study, a piezoelectric fast-steeringmirror (FSM) is considered to provide accurate pointing to $\mu \mathrm{rad}$ level. Via a hardware-based approach, the piezoelectric FSM is comprehensively simulated including both linear and nonlinear hysteresis parts. The ratedependent characteristic of the piezoelectric hysteresis is modeled and its effect on the final control accuracy will be shown. Furthermore, based on the hysteresis model, an inverse controller is presented. A combination of the inverse controller and a traditional PID one suppresses the effect of the hysteresis and thus, improves the control performance.

\section{OPEN-LOOP POINTING BUDGET AND LINK BUDGET ANALYSIS}

\subsection{Open-loop Pointing Budget}

To establish a communication link between a 100-kg class satellite and its GEO counterpart, the small satellite must initially transmit a laser beacon to search for the GEO satellite's position. This process can be implemented by either sending a wide laser beam or scanning a narrow one from the LEO satellite. In the present study, to keep the optical control system of the small satellite simple, the former option was selected. Accordingly, the small LEO satellite points a wide beam toward the predicted position of the GEO terminal by using its attitude determination and control system. Once detecting the beacon, the GEO satellite will transmit back a narrow beam to the small satellite in LEO orbit. Similarly, the LEO satellite predicts the GEO terminal based on the received narrow laser beam and transmits another narrow beam back to track the GEO satellite. When both optical systems of the two satellites lock to each other, data transmission can be started. During the initial phase, generally, there are three main error sources that make the beacon light off the target (see Fig. 1). The first one is the error of the orbit information that the small satellite uses to initially determine the position of the GEO satellite. Another main source comes from the pointing capability of the LEO satellite. The body pointing accuracy of a 100-kg class satellite can be of the order of $100 \mu \mathrm{rad}$ to several hundred $\mu \mathrm{rad} .{ }^{5,6}$ In this study, the 


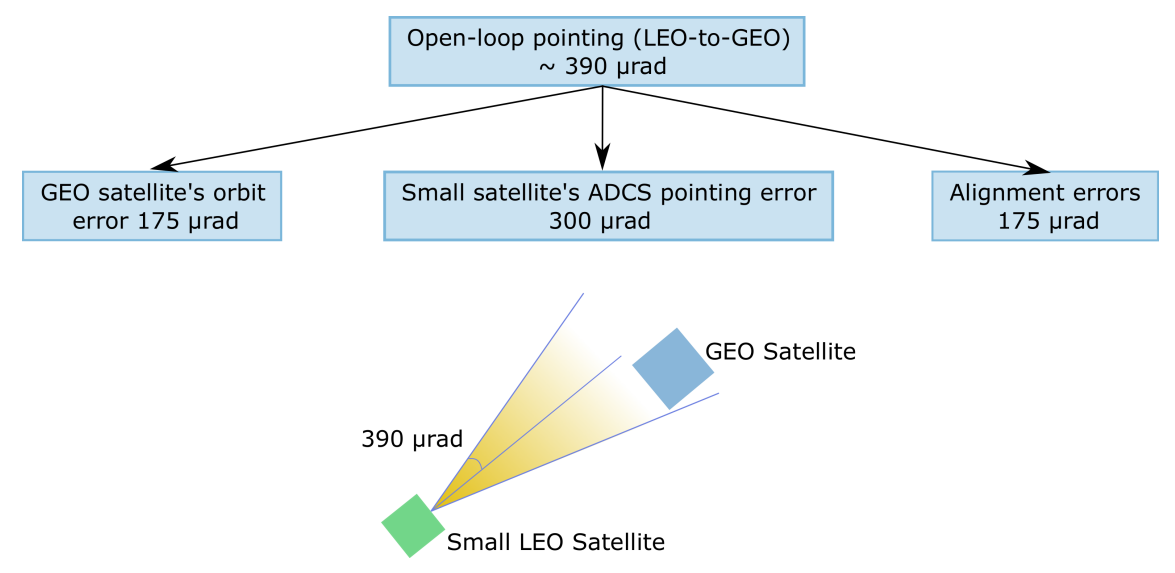

Figure 1: Open-loop pointing budget.

value of $300 \mu \mathrm{rad}$ is taken as a reference for the attitude control accuracy of the LEO satellite. Finally, the third source is the misalignment errors constituted by the environmental and calibration errors. Adding the three main sources as the root sum square (RSS), it can be concluded that a 780- $\mu$ rad beacon beam transmitted from the LEO satellite is able to initially cover the uncertainty of the GEO satellite's position so that the communication link can be established.

\subsection{Link Budget Analysis}

In order to define a practical set of link system parameters, state-of-the-art laser communication technologies of small satellites are investigated. Recently, a laser transmitter that can provide up to 4-10 W output power was reported in the Aerocube- 7 project. $^{7}$ Therefore, a $5-\mathrm{W}$ transmitting power is assumed for the LEO-toGEO communication link. Due to extremely narrow laser beams, the pointing accuracy requirement must achieve a $\mu \mathrm{rad}$ level. To do that, the 100-kg class satellite's body pointing is combined with a fine pointing mechanism (FPM) using a piezoelectric FSM. Compared to other mechanisms such as voice-coils and microelectro-mechanical systems (MEMS), the piezoelectric actuator shows its advantages of achieving very high control accuracy, fast response and satisfying the SWaP constraints. As for the counterpart of the small LEO satellite, the National Institute of Communications and Information Technology, Japan, has been developing the GEO ETS-XI satellite equipped with High Speed Communication with Advanced Laser Instrument (HICALI). 8,9 With a 15-cm aperture and high receiver sensitivity, HICALI is suitable to be a relay node of the small satellite because it can compensate for the lack of the small satellite's resources. Based on the existing technologies, a link budget of the LEO-to-GEO laser communication is calculated for both the initial and communication phases. Using the link budget tool, the dependency of the link margin on the data rate and final pointing accuracy, two important trade-off parameters, is shown in Fig. 2.

In order to achieve a 3 - $\mathrm{dB}$ link margin, the required pointing accuracy for the relay communication is $10 \mu \mathrm{rad}$ and the corresponding data rate is $300 \mathrm{Mbps}$. With approximate 45 minutes for each communication session, a considerably larger amount of data can be transmitted from the small LEO satellite to the GEO terminal. ${ }^{10}$ The results of the beacon and communication link calculation are summarized in Tab. 2.

\section{PIEZOELECTRIC FAST-STEERING-MIRROR MODELING}

\subsection{Pointing, Acquisition, and Tracking System}

In principle, to achieve the control accuracy of $10 \mu \mathrm{rad}$ as derived in Sec. 2.2, the pointing-acquisition-tracking system of the small satellite must contain two stages. While a coarse control stage directs laser beams coarsely to the GEO terminal, a FPM system points them accurately to track and communicate with the GEO counterpart. Due to the SWaP constraints, the 100-kg class satellite's body pointing is utilized as the coarse control stage instead of bulky gimbal systems as in past projects. The 300- $\mu$ rad coarse pointing error can then be compensated by the FPM system. In the FPM system, a piezoelectric FSM was chosen to direct laser beams accurately with 


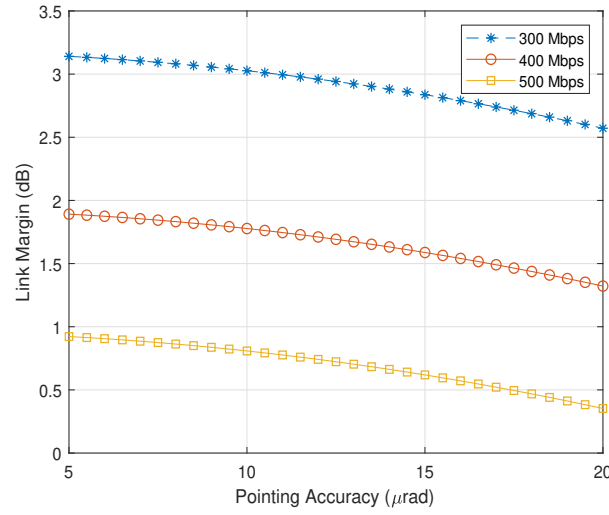

Figure 2: The dependency of the link margin on the data rate and pointing accuracy.
Table 2: Beacon and communication link budgets of the LEO-to-GEO transmission.

\begin{tabular}{|l|c|c|c|}
\hline Item & Beacon & Comm & Unit \\
\hline Transmit power & 4 & 5 & $\mathrm{~W}$ \\
\hline Wavelength & 1.55 & 1.55 & $\mu \mathrm{m}$ \\
\hline $\begin{array}{l}\text { LEO } T_{x} \text { antenna } \\
\text { diameter }\end{array}$ & 5 & 5 & $\mathrm{~cm}$ \\
\hline Strehl ratio & 0.4 & 0.4 & $\mathrm{~dB}$ \\
\hline Pointing accuracy & 300 & 10 & $\mu \mathrm{rad}$ \\
\hline Distance & 44,000 & 42,000 & $\mathrm{~km}$ \\
\hline $\begin{array}{l}\text { GEO } R_{x} \text { antenna } \\
\text { diameter }\end{array}$ & 15 & 15 & $\mathrm{~cm}$ \\
\hline Margin & + & + & $\mathrm{dB}$ \\
\hline
\end{tabular}

a high speed and fine resolution. Nevertheless, the control accuracy of the piezoelectric actuator is severely affected by hysteresis phenomenon. Thus, it is necessary that the hysteresis must be modeled accurately so that a proper controller can be derived to suppress it. In the following, a piezoelectric FSM that is suitable for the LEO-to-GEO communication mission is comprehensively modeled including both a linear part and a hysteresis part.

\subsection{Experiment setup}

A piezoelectric FSM manufactured by Core Tomorrow Science \& Technology company was considered for the LEO-to-GEO laser communication mission. With a nominal travel angle range up to $2.5 \mathrm{mrad}$, the FSM can fully cover the residual error of the small satellite's pointing. Additionally, it has a very-high resolution to help realizing the final control accuracy at the $\mu \mathrm{rad}$ level. In order to comprehensively simulate the piezoelectric FSM, an experiment was conducted. Analog control signals generated by a functional generator were sent to the controller through which the signals were magnified to excite the piezoelectric FSM. The amplification ratio of the controller is 15 . The displacement of the piezoelectric actuator was measured by a strain gauge sensor (SGS). Both the analog control signals from the functional generator and the SGS's signals were discretized and recorded in a computer by using a National Instruments (NI) USB-6343 card and LabVIEW program for the purpose of simulating the piezoelectric FSM. The sample frequency was set to $50 \mathrm{Khz}$. The setup of the experiment is described in Fig. 3.

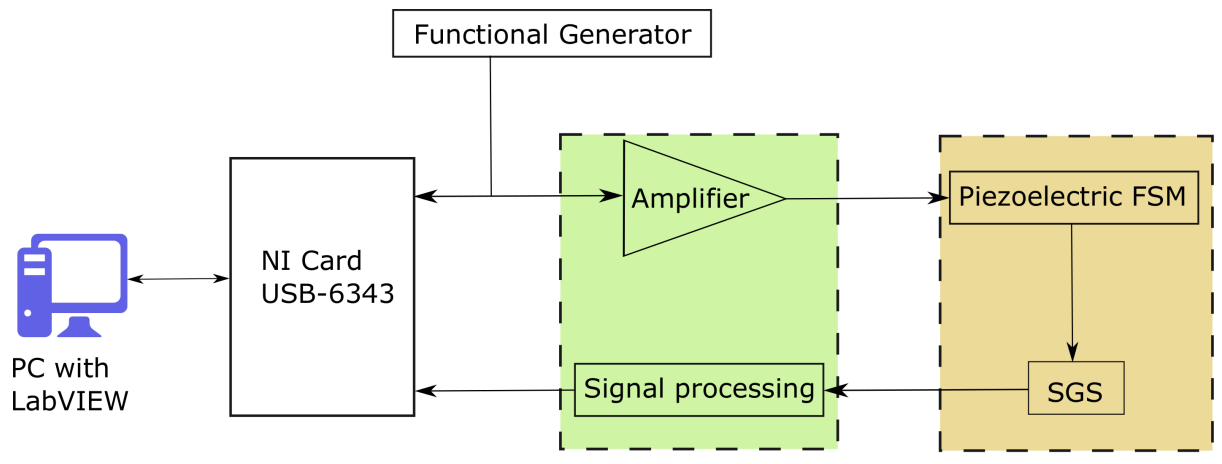

Figure 3: Block diagram of the experiment to collect data for modeling. 


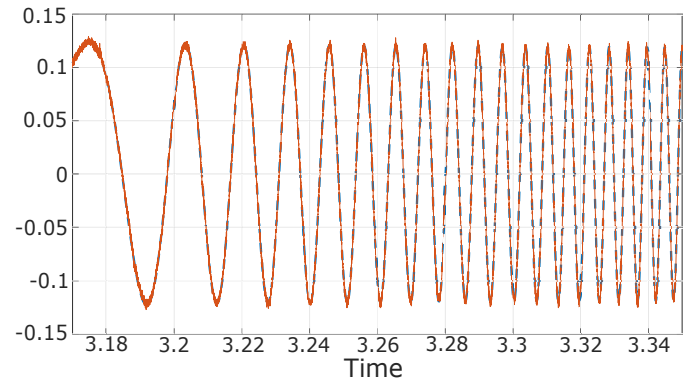

(a) Output responses
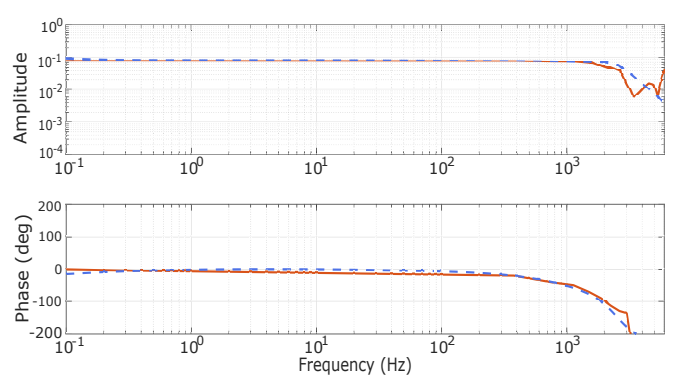

(b) Frequency responses

Figure 4: Comparison of the simulated output response of the identified linear dynamics and output response measurement.

\subsection{Modeling linear dynamics and nonlinear hysteresis of the piezoelectric FSM}

A comprehensive model of the piezoelectric FSM is divided into two parts: a linear dynamic part and a nonlinear (hysteresis) one. Considering both electric and mechanical dynamics, the transfer function of the linear dynamic part of the piezoelectric FSM can be expressed as:

$$
G_{\text {linear }}(s)=\frac{a_{2} s^{2}+a_{1} s+a_{0}}{s^{5}+b_{4} s^{4}+b_{3} s^{3}+b_{2} s^{2}+b_{1} s+b_{0}} .
$$

To identify the coefficients of the transfer function in Eq. 1, a sinusoidal chirp signal of increasing frequency was generated to excite the piezo-actuated stage in an open-loop configuration. The amplitude of the chirp signal was set to be small to reduce the effect of hysteresis nonlinearity. Additionally, the 50-Khz sampling frequency is sufficiently high to avoid the creep behaviour. In the experiment, LabVIEW program was used to record the input chirp and the corresponding SGS's signals in a host computer. Then, based on the stored data, a system identification method was implemented in Matlab/Simulink to obtain the transfer function of the linear dynamic part of the piezoelectric FSM:

$$
G_{\text {linear }}(s)=\frac{1.979 \times 10^{11} s^{2}+7.051 \times 10^{13} s+2.973 \times 10^{13}}{s^{5}+2.15 \times 10^{4} s^{4}+3.737 \times 10^{8} s^{3}+2.615 \times 10^{12} s^{2}+8.489 \times 10^{14} s+1.829 \times 10^{14}} .
$$

The simulated and measured outputs of the linear dynamic part of the piezoelectric FSM are presented in Figs. 4. The fitting level between the simulation and measurement data is $97 \%$ approximately, which validates the linear dynamics model of the FSM.

Although a piezoelectric actuator shows its advantages in providing a very high control accuracy, it suffers from the effect of hysteresis. Due to the natural nonlinear characteristic of the hysteresis, it is difficult to use traditional methods to control the piezoelectric actuator accurately. Consequently, modeling the piezoelectric hysteresis is of interest of many researchers to design proper controllers and suppress the nonlinear effect. Among many models that have been studied the hysteresis behavior, the Bouc-wen model is attractive due to its simplicity in numerical implementation and ability to describe many forms of hysteresis loops. In the present LEO-to-GEO laser communication model, it is desirable to operate the piezoelectric FSM of the FPM system with a high speed to suppress the effect of satellite's vibration. ${ }^{11,12}$ Nevertheless, it is a well-known fact that the higher the rate of control signals is, the more serious the hysteresis nonlinearity becomes. Therefore, for the purpose of designing a proper controller, the rate-dependent characteristic of the piezoelectric hysteresis must be modeled accurately. A modified Bouc-wen model that can capture the rate-dependent behavior of the hysteresis can be described as: ${ }^{13}$

$$
\begin{aligned}
& y(t)=z(t)+h(t)=d_{1} e^{-d_{2} \dot{u}(t)} u(t)+h(t) \\
& \dot{h}(t)=\epsilon e^{\delta \mid \dot{u}(t)} \dot{u}(t)-\beta \dot{u}(t)|h(t)|-\alpha|\dot{u}(t)| h(t)
\end{aligned}
$$




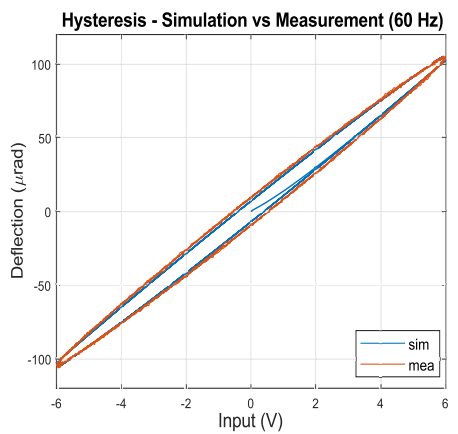

(a) $60 \mathrm{~Hz}$

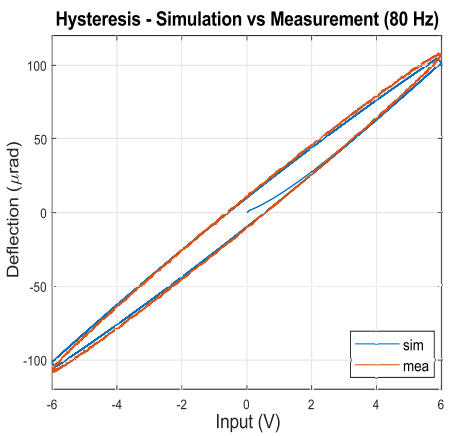

(b) $80 \mathrm{~Hz}$

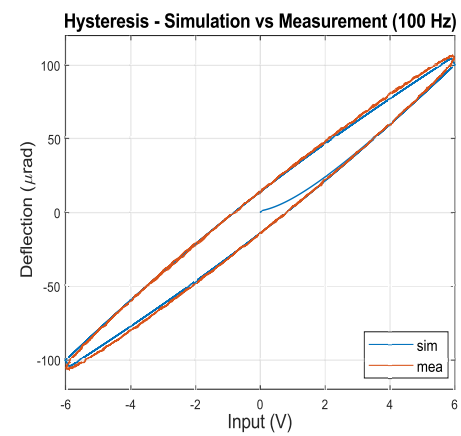

(c) $100 \mathrm{~Hz}$

Figure 5: The rate-dependent hysteresis of the piezoelectric FSM with the input frequency as $60 \mathrm{~Hz}, 80 \mathrm{~Hz}$, and 100 $\mathrm{Hz}$, respectively.

where, $u(t)$ is the excited signal of the stage, $y(t)$ is the corresponding displacement of the piezoelectric material of the actuator, and $h(t)$ is the hysteresis. $d_{1}, d_{2} \epsilon, \delta, \beta, \alpha$ are the parameters of the hysteresis model and they can be identified by using data obtained in experimental trials. Accordingly, a set of sinusoidal signals $u(t)=6 \sin (2 \pi f)+$ $6(\mathrm{~V})$ with different frequencies $f$ were applied to excite the FSM. The control and corresponding displacement signals were then recorded. Based on the obtained data of the $100-\mathrm{Hz}$ control signal, Matlab/Simulink software was again used for system identification. The nonlinear least square method using Trust-Region-Reflective algorithm was implemented to identify the parameters of the nonlinear hysteresis model. The results of the system identification are: $d_{1}=0.089, d_{2}=-2.96 \times 10^{-7}, \epsilon=-0.048, \delta=0.00047, \beta=34.635$, and $\alpha=38.421$. The identified hysteresis was then compared with measured data for different input frequencies $(f=60 \mathrm{~Hz}$ and $80 \mathrm{~Hz}$ ). As can be seen in Fig. 5, the hysteresis model agrees well with the measurement results. This indicates that the identified model can efficiently describe the rate-dependent characteristic of the hysteresis of the piezoelectric FSM.

\section{A COMBINATION OF FEEDFORWARD AND FEEDBACK CONTROL TO SUPPRESS THE EFFECT OF HYSTERESIS ON CONTROL ACCURACY}

In this section, the comprehensive identified model of the piezoelectric FSM was used in a simulation to verify its control accuracy. At first, the traditional PID control method was used to drive the piezoelectric FSM to track two reference sinusoidal signals whose both amplitudes are $300 \mu \mathrm{rad}$ and frequencies are $1 \mathrm{~Hz}$ and $15 \mathrm{~Hz}$,

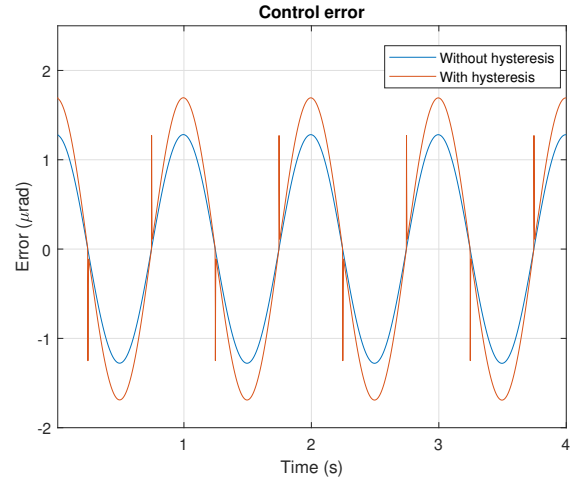

(a) $1 \mathrm{~Hz}$

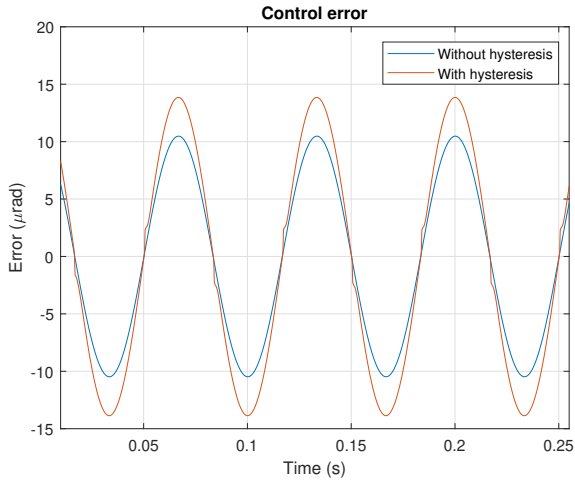

(b) $15 \mathrm{~Hz}$

Figure 6: Tracking errors of the PID controller with 1-Hz and 15- Hz input frequencies. 


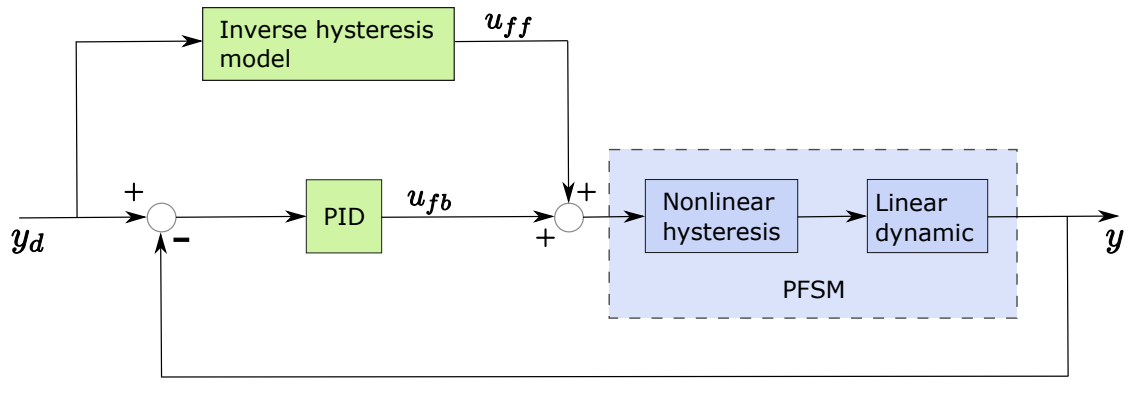

Figure 7: The block diagram of the combined feedforward and feedback control scheme.

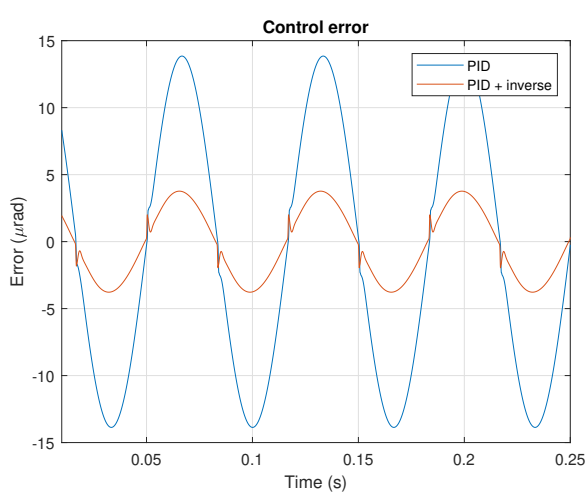

(a) Tracking error $(15 \mathrm{~Hz})$

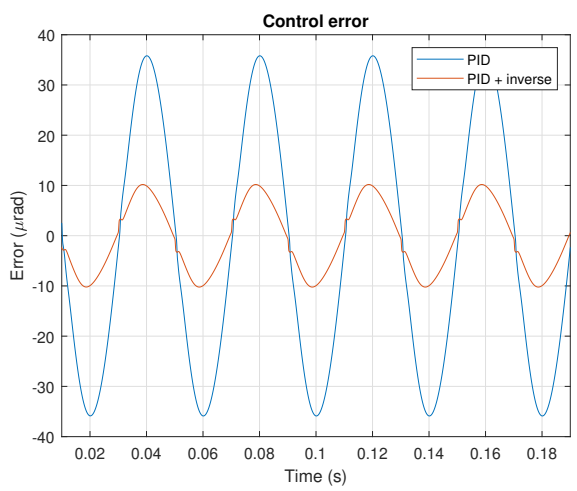

(c) Tracking error $(25 \mathrm{~Hz})$

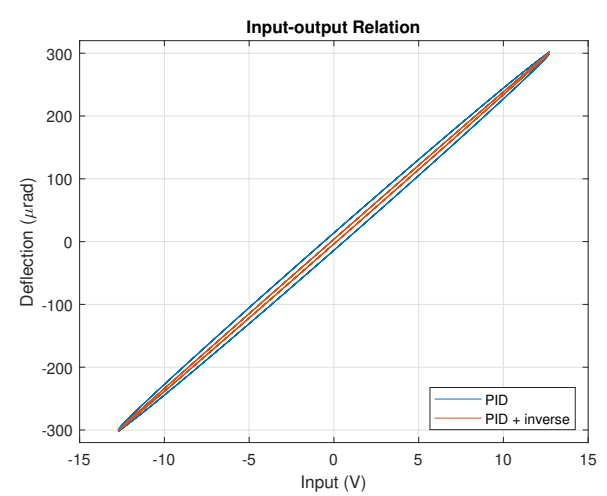

(b) Input-output relation $(15 \mathrm{~Hz})$

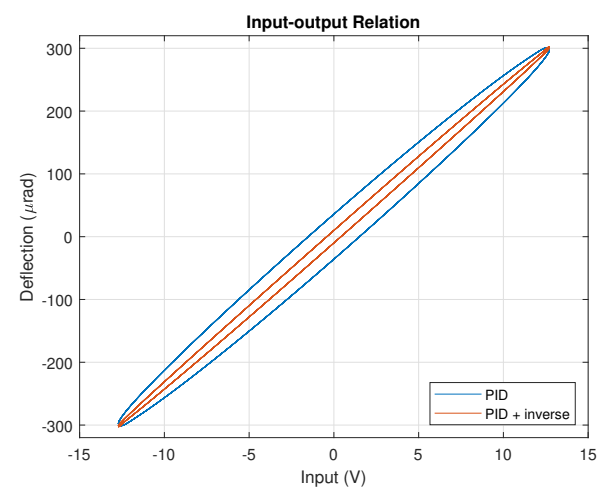

(d) Input-output relation $(25 \mathrm{~Hz})$

Figure 8: Comparison of the control performance between the PID controller and combined controller with $15-\mathrm{Hz}$ and $25-\mathrm{Hz}$ input frequencies.

respectively. The tracking errors of the simulation with and without the hysteresis are shown in Fig. 6 . It is clearly seen that due to the hysteresis nonlinearity, the tracking errors become worse, especially when the driving frequency increases. To improve tracking error in hysteretic systems, feedforward control is often combined with feedback control. ${ }^{14,15}$ Therefore, a combination of the PID feedback controller and a feedforward controller is proposed to suppress the effect of the nonlinear hysteresis and thus, improve the control performance. The feedforward mechanism is based on the inverse multiplicative-parameter method proposed in Ref. 16. Hence, the inverse controller can be simply derived based on the hysteresis model identified in Sec. 3.3. A block diagram of the fine control stage with the combination of the feedforward and feedback control scheme is shown in Fig. 7 . 
In Eq. 3, assuming the input reference is $y_{d}(t)$ and the hysteresis component is identified as in Sec. 3.3, the output voltage $u_{f f}$ of the feedforwad controller can be expressed as:

$$
u_{f f}(t)=\frac{y_{d}(t)-h(t)}{d_{1} e^{-d_{2} \dot{u}(t)}} .
$$

The output $u_{f f}$ of the inverse model-based feedforward controller is then combined with the output $u_{f b}$ of the PID controller to drive the piezoelectric FSM to track input references. The efficiency of the proposed controller was investigated with two $15-\mathrm{Hz}$ and $25-\mathrm{Hz}$ sinusoidal reference signals. As being shown in Fig. 8, compared to the PID controller, the combined controller significantly improves the tracking error. The error decreases of about $10 \mu \mathrm{rad}$ and $25 \mu \mathrm{rad}$ in two cases, respectively. These results indicate the efficiency of the combination of the PID and inverse controllers in suppressing the nonlinear hysteresis and thus, improving the control performance.

\section{CONCLUSION}

In this paper, the concept in which a 100-kg class satellite transmits a high-speed laser link to a GEO terminal has been introduced. In order to realize the LEO-to-GEO laser link, open-loop pointing and communication link budgets were conducted to propose a practical set of link system parameters. The result can be utilized for further detailed designs in the future. To achieve the stringent pointing accuracy requirement, the LEO satellite's attitude control is combined with a fine control stage using a piezoelectric FSM. The FSM was comprehensively modeled including both the rate-dependent nonlinear hysteresis part and linear dynamic part. Based on the hysteresis model, an inverse controller was presented to work as a feedforward mechanism together with a feedback PID controller. The combination of the feedforward and feedback control scheme has improved the control performance, especially when rate of input control signals is high. Extension simulation that take account of vibration disturbance and noise of feedback sensors is regarded as future work.

\section{ACKNOWLEDGMENTS}

The authors would like to thank the Global Leader Program for Social Design and Management (GSDM) of the University of Tokyo and Vietnam National Space Center (VNSC), under Vietnam Academy of Science and Technology (VAST) for their support during this study.

\section{REFERENCES}

[1] Obata, T., Saito, H., Tanaka, K., Nakasuka, S., and Shirasaka, S., "The development status of the first demonstration satellite of our commercial small synthetic aperture radar satellite constellation," in [Small Satellite Conference], (2019).

[2] Tolker-Nielsen, T. and Oppenhauser, G., "In-orbit test result of an operational optical intersatellite link between ARTEMIS and SPOT4, SILEX," in [Free-Space Laser Communication Technologies XIV], Mecherle, G. S., ed., 4635, 1 - 15, International Society for Optics and Photonics, SPIE (2002).

[3] Jono, T., Takayama, Y., Shiratama, K., Mase, I., Demelenne, B., Sodnik, Z., Bird, A., Toyoshima, M., Kunimori, H., Giggenbach, D., Perlot, N., Knapek, M., and Arai, K., "Overview of the inter-orbit and the orbit-to-ground laser communication demonstration by OICETS," in [Free-Space Laser Communication Technologies XIX and Atmospheric Propagation of Electromagnetic Waves], Mecherle, S. and Korotkova, O., eds., 6457, 9 - 18, International Society for Optics and Photonics, SPIE (2007).

[4] Hauschildt, H., Mezzasoma, S., Moeller, H. L., Witting, M., and Herrmann, J., "European data relay system goes global," in [2017 IEEE International Conference on Space Optical Systems and Applications (ICSOS)], 15-18 (2017).

[5] Ilsen, S., Gerrits, D., Vrancken, D., Naudet, J., Mellab, K., Santandrea, S., Laroche, T., and Verheyden, A., "Proba-v: The example of onboard and onground autonomy," in [Small Satellite Conference], (2014).

[6] Fuchs, C., Moll, F., Giggenbach, D., Schmidt, C., Keim, J., and Gaisser, S., "Osirisv1 on flying laptop: Measurement results and outlook," in [2019 IEEE International Conference on Space Optical Systems and Applications (ICSOS)], 1-5 (2019). 
[7] Rose, T. S., Rowen, D. W., Coffman, C. M., Kinum, G., LaLumondiere, S., Werner, N. I., Wicker, J., Maul, G. A., and Welle, R. P., "Optical downlink and intersatellite illumination experiments with low-earth orbiting cubesats," in [Free-Space Laser Communications XXXII], Hemmati, H. and Boroson, D. M., eds., 11272, 369 - 374, International Society for Optics and Photonics, SPIE (2020).

[8] Toyoshima, M., "Recent trends in space laser communications for small satellites and constellations," in [2019 IEEE International Conference on Space Optical Systems and Applications (ICSOS)], 1-5 (2019).

[9] Carrasco-Casado, A., Do, P. X., Kolev, D., Hosonuma, T., Shiratama, K., Kunimori, H., Trinh, P. V., Abe, Y., Nakasuka, S., and Toyoshima, M., "Intersatellite-link demonstration mission between cubesota (leo cubesat) and ets9-hicali (geo satellite)," in [2019 IEEE International Conference on Space Optical Systems and Applications (ICSOS)], 1-5 (2019).

[10] Do, P. X., Carrasco-Casado, A., Hosonuma, T., Toyoshima, M., and Nakasuka, S., "A study on optimizing laser beam waist for leo-to-geo communication for $100 \mathrm{~kg}$-class satellite," in [2020 International Conference on Communications, Computing, Cybersecurity, and Informatics (CCCI)], 1-6 (2020).

[11] Toyoshima, M., Takayama, Y., Kunimori, H., Jono, T., and Yamakawa, S., "In-orbit measurements of spacecraft microvibrations for satellite laser communication links," Optical Engineering 49(8), 1 - 10 (2010).

[12] Haghshenas, J., "Maximum allowable low-frequency platform vibrations in high resolution satellite missions: challenges and look-up figures," in [Optical Systems Design 2015: Optical Design and Engineering VI], Mazuray, L., Wartmann, R., and Wood, A. P., eds., 9626, 740 - 749, International Society for Optics and Photonics, SPIE (2015).

[13] Gan, J. and Zhang, X., "Nonlinear hysteresis modeling of piezoelectric actuators using a generalized bouc-wen model," Micromachines 10, 183 (Mar 2019).

[14] Hassani, V., Tjahjowidodo, T., and Do, T. N., "A survey on hysteresis modeling, identification and control," Mechanical Systems and Signal Processing 49(1), 209-233 (2014).

[15] Fang, J., Wang, J., Li, C., Zhong, W., and Long, Z., "A compound control based on the piezo-actuated stage with bouc-wen model," Micromachines 10, 861 (Dec 2019).

[16] Rakotondrabe, M., "Bouc-wen modeling and inverse multiplicative structure to compensate hysteresis nonlinearity in piezoelectric actuators," IEEE Transactions on Automation Science and Engineering 8(2), 428-431 (2011). 